\title{
La hacienda Huanquisco Canta en manos de la familia Mendoza Iturri, provincia Omasuyos, La Paz-Bolivia
}

\author{
The Hacienda Huanquisco Sings in the hands of the Mendoza Iturri \\ family, Omasuyos Province, La Paz-Bolivia \\ A Hacienda Huanquisco Canta nas mãos da família Mendoza Iturri, \\ Omasuyos Province, La Paz-Bolivia
}

Giovana Alba Ninachoque Quispe ${ }^{1}$

Instituto Mora, México

Para citaciones: Ninachoque, G. (2021). La hacienda Huanquisco Canta en manos de la familia Mendoza Iturri, provincia Omasuyos, La Paz-Bolivia. El Taller de la Historia, 13(1), 151-170.

Recibido: marzo 2021

Aprobado: junio de 2021

Editor: Sergio Paolo Solano. Universidad de Cartagena-Colombia.
Copyright: $\odot$ 2021. Ninachoque, G. Este es un artículo de acceso abierto, distribuido bajo los términos de la licencia https://creativecommons.org/licenses/by-nc$\mathrm{sa} / 4.0 /$ la cual permite el uso sin restricciones, distribución y reproducción en cualquier medio, siempre y cuando que el original, el autor y la fuente sean acreditados.

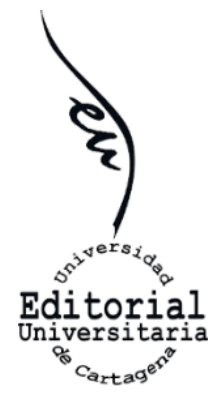

\section{RESUMEN}

El presente trabajo tiene como propósito analizar el estudio particular e introductorio de la hacienda Huanquisco Canta, situada en el cantón de Ancoraimes, provincia de Omasuyos, departamento de La Paz, que estuvo en manos del hacendado Benjamín Mendoza Iturri, procedente de una élite terrateniente muy importante en este sector del territorio nacional. A partir de este estudio se busca recuperar la historia de los pueblos indígenas que quedaron en el silencio y el olvido, por obra de aquella élite terrateniente la de los Mendoza Iturri, que controló diversos pisos ecológicos como altiplano y valle, hecho que se reflejó en el gran control local que llego a tener gracias a la herencia en tierras que recibieron en los espacios de Achacachi, Ancoraimes y Tímusi. Respecto a las fuentes hemos y nos sustentamos en padrones republicanos, expedientes agrarios y bibliografía especializada sobre el tema.

Palabras clave: Hacienda; élite terrateniente; tierras comunales; comunidad indígena; reforma agraria.

\begin{abstract}
The present work aims to analyze the particular and introductory study of the Huanquisco Canta farm, located in the canton of Ancoraimes, Omasuyos province, La Paz department, which was in the hands of the landowner Benjamín Mendoza Iturri, from a very elite landowner important in this sector of the national territory. From this study it is sought to recover the history of the indigenous peoples who were left in silence and oblivion, by the work of that landowning elite, the Mendoza Iturri, who controlled various ecological floors such as plateau and valley, a fact that was reflected in the great local control that I came to have thanks to the inheritance in lands that they received in the spaces of Achacachi, Ancoraimes and Tímusi. Regarding the sources, we have and are based on republican registers, agrarian records and specialized bibliography on the subject.
\end{abstract}

\footnotetext{
${ }^{1}$ maestría en Historia, Universidad Andina Simón Bolívar. Doctorante en Historia Moderna y Contemporánea del Instituto
} Mora, México. albagiovana@gmail.com 
Keywords: Finance; landed elite; communal lands; indigenous community; agrarian reform.

\section{RESUMO}

O objetivo deste artigo é analisar o estudo particular e introdutório da fazenda Huanquisco Canta, localizada no cantão de Ancoraimes, província de Omasuyos, departamento de La Paz, que estava nas mãos do proprietário Benjam Mendoza Iturri, de uma elite de terras muito importante neste setor do território nacional. A partir deste estudo buscamos recuperar a história dos povos indígenas que foram deixados em silêncio e esquecimento, pelo trabalho daquela elite proprietária do Mendoza Iturri, que controlava vários andares ecológicos como altiplano e vale, fato que se refletiu no grande controle local que passou a ter graças à herança em terras que receberam nos espaços de Achacachi, Ancoraimes e Tímusi. Em relação às fontes, temos e contamos com registros republicanos, registros agrários e bibliografia especializada sobre o assunto.

Palavras-chave: Finanças; elite proprietário de terra; terras comunitárias; comunidade indígena; reforma agrária.

\section{Introducción}

La historia del área rural y de los pueblos indígenas bolivianos es poca conocida y difundida. Dentro de los intereses de nuestros gobiernos no se encontraban los pueblos indígenas y, menos aún, su historia, motivo por el cual los trabajos de investigación sobre este tema son escasos en estos últimos tiempos. El problema agrario indígena boliviano data del periodo colonial, y continuo más allá de la mitad del siglo XX. Las políticas de los gobiernos conservadores y liberales intentaron resolver la cuestión de la tenencia de la tierra a través de la distribución individual entre indígenas originarios y forasteros. José Carlos Mariátegui explicó en su época que "el problema del indio es el problema de la tierra". A lo largo del siglo XIX y la primera mitad del siglo XX, los gobiernos de turno expoliaron la tierra a los indígenas para sostener el gamonalismo mediante la hacienda y el latifundio ${ }^{2}$.

El nacimiento de la hacienda en el periodo colonial tiene su origen en la encomienda y la reducción de pueblos indígenas, durante el periodo toledano. Desde los siglos XVII y XVIII se produce un lento proceso de avance de la hacienda colonial. En cambio, en el periodo republicano se consolida un periodo caótico para las comunidades indígenas, debido a las políticas agrarias establecidas en los gobiernos de Mariano Melgarejo (1864-1871) y Tomas Frías (1872-1874). Durante el gobierno del primero se promulgaron

\footnotetext{
${ }^{2}$ José Carlos Mariátegui, 7 ensayos de interpretación de la realidad peruana, Caracas, Fundación Biblioteca Ayacucho, 2007.
} 
una infinidad de decretos supremos y leyes agrarias, siendo muy importante el decreto del 20 de marzo de 1866.

Artículo $2^{\circ}$ : Cada indígena de los expresados deberá obtener del Gobierno Supremo el título de su propiedad particular, previo abono de una cantidad que no pasara de veinticinco pesos [...] Artículo $4^{\circ}$ : El indígena que dentro el término de sesenta días, después de ser notificado, no recabare el expresado título, el terreno será enajenado en pública subasta.

Con la implementación de dicho decreto se establecía la venta de las tierras de comunidad, lo que significó a la postre el despojo a los indígenas. Posteriormente, durante el gobierno de Tomas Frías también se promulgaron una serie de leyes y decretos en contra de las tierras de comunidad. Tal es el caso de la Ley del 5 de octubre de 1874 que ordenaba la ex-vinculación de tierras de origen, conocida propiamente como la revisita de tierras. El principal objetivo de esta ley fue establecer la propiedad privada individual de la tierra y desconocer por completo la propiedad de las comunidades indígenas.

Artículo $10^{\circ}$ : El supremo gobierno mandará practicar la revisita general de cada provincia, por una comisión revisitadora compuesta de un revisitador, del subprefecto, de un secretario, de un perito agrimensor titulado y del párroco en cada cantón [...] Artículo $27^{\circ}$ : los indígenas que no hubiesen obtenido el título de sus tierras y sus herederos y demás descendientes, no podrán e ningún tiempo ser matriculados por razón de la contribución personal y no estarán sujetos sino a pagar los impuestos que pesaren los bolivianos en general [...] Artículo $28^{\circ}$ : concluida la revisita en cada provincia, quedará prohibida por completo, la matrícula de los indígenas sin tierras, para el pago de la contribución personal.

Si bien la Ley de ex-vinculación declaró el derecho de propiedad a los indígenas que poseían tierras, también se declaró propiedad del Estado a las tierras sobrantes, pues, a su vez, dicha legislación ordenaba practicar la revisita de tierras en todas las provincias del altiplano y valle.

El presente trabajo, está enfocado en el estudio particular e introductorio de la hacienda Huanquisco Canta, situada en el cantón de Ancoraimes, provincia de Omasuyos, departamento de La Paz, que estuvo en manos del hacendado Benjamín Mendoza Iturri, procedente de una élite terrateniente muy importante en este sector del territorio nacional.

Así mismo, se busca recuperar la historia de los pueblos indígenas que quedaron en el silencio y el olvido, por obra de aquella élite terrateniente la de los Mendoza Iturri, que controló los espacios de Achacachi, Ancoraimes y Tímusi. 


\section{Formación y consolidación de haciendas en la provincia de Omasuyos, departamento de La Paz}

El proceso y formación de las haciendas y venta de las tierras comunales, no fue solo producto de la Ley de 1866. Entre 1881 y 1920, la venta de tierras de comunidad en el departamento de La Paz se dio en dos períodos según Erwin P. Grieshaber, el primer periodo de venta de tierras se da en gran magnitud entre 1881 y 1886 representando un total del $40 \%$ de venta de tierras, mientras el segundo periodo corresponde y entre 1905 y 1915 con un porcentaje de un $38 \%{ }^{3}$. Los datos expresados manifiestan que hay una disminución del $2 \%$ en la venta de tierras comunales para el segundo periodo. Esta disminución, a nuestro parecer, se debe a que, en la primera etapa, en todo el altiplano boliviano las comunidades indígenas, casi en su mayoría, fueron convertidas en haciendas. Por esta razón, la venta de tierras disminuye en la segunda etapa, y además, como dice Erwin Grieshaber, las personas relacionadas con la Guerra Federal de 1899 y con el Partido Liberal, fueron las que adquirieron mayor cantidad de tierras de comunidad.

Es así, que la venta de tierras en el departamento de La Paz fue muy rápida especialmente en aquellas zonas cercanas a la ciudad de La Paz. Particularmente fueron aquellas tierras que tenían mejores condiciones de productividad agropecuaria: ubicadas cerca del Lago Titicaca o aquellas que estaban ubicadas en los valles altiplánicos ${ }^{4}$.

Bajo este panorama, la provincia de Omasuyos fue una de las regiones donde se practicaron tempranamente las reducciones que impuso el virrey Francisco de Toledo y una de las 16 provincias que aportó con mitayos a Potosí para el trabajo en las minas, lo que constituyó uno de los factores de la disminución de la población ${ }^{5}$. Pero al mismo tiempo, la particularidad de sus suelos y clima dio origen al nacimiento de haciendas, desde el siglo XVII, tal como lo señala Antonio Rojas:

Omasuyos representa quizás el espacio económico y social más patético en los avatares de la contradictoria historia del altiplano boliviano. Era ya reconocida por la sociedad colonial como una región privilegiada por las peculiaridades del medio ecológico donde se encuentra: pampa "rinconada" y laderas de la cordillera occidental se extienden bordeando el lago Titicaca beneficiándose del micro clima favorable, en la parte oriental de su extensión, de más de $200 \mathrm{Km}^{26}$.

\footnotetext{
${ }^{3}$ Erwin P. Grieshaber, "Resistencia indígena a la venta de tierras comunales en el departamento de La Paz, 1881-1920". Data, 1, 1991.

${ }^{4}$ Herbet Klein, Haciendas y Ayllus, siglos XVIII y XIX, Lima, IEP, 1995, p. 192.

${ }^{5}$ A propósito de la instauración de la mita, van a aparecer los llamados "forasteros" o "agregados" que se dirigen a distintas regiones incorporándose a las comunidades indígenas. Por ello, los términos forastero o agregado se refieren a los descendientes de los comunarios que entraron en las comunidades desde el exterior, después de dejar sus tierras de origen a causa de epidemias o trabajo forzado, para buscar nuevos espacios en otras comunidades fuera de sus lugares de origen. Véase a Froilán Mamani Humerez, "La práctica del arrendamiento durante el siglo XIX en la región de Santiago de Huata", en María Luisa Soux, (coord), El proceso histórico hacia la territorialización del poder, La Paz, Instituto de Estudios Bolivianos, 2012, p. 41.

${ }^{6}$ Antonio Rojas, "La tierra y el trabajo en la articulación de la economía campesina con la hacienda". Avances, $2,1978: 52$.
} 
El periodo de expansión de haciendas en la provincia de Omasuyos data desde el tardío siglo XVIII. Herbert Klein en su estudio Haciendas y Ayllus en Bolivia, siglos XVII y XIX, nos proporciona datos interesantes sobre la expansión de las haciendas en dicha provincia hasta el siglo XIX.

La mayoría de la población campesina india de la región estaba distribuida entre 491 comunidades indias libres -o ayllus- poseedores de tierras. Las 1,099 haciendas poseídas por 721 haciendas, comprendían solo unos 83,000 trabajadores campesinos, o alrededor del $40 \%$ del total provincial y sin embargo eran el elemento dominante en la producción comercial agrícola ${ }^{7}$.

En la tabla 1, podemos ver con más detalle la consolidación de las primeras haciendas desde fines del periodo colonial en Omasuyos, Chulumani, Sicasica, Larecaja, Pacajes y La Paz, resaltando principalmente el número de haciendas, yanaconas y el total de la población india registrados en la provincia de Omasuyos, en los censos de 1786 y 1797.

Tabla 1: Haciendas y yanaconas en la intendencia de La Paz entre 1786-1797

\begin{tabular}{|l|c|c|c|c|}
\hline \multicolumn{1}{|c|}{ Distritos } & $\begin{array}{c}\text { Fecha del } \\
\text { censo }\end{array}$ & $\begin{array}{c}\mathbf{N}^{\circ} \text { de } \\
\text { haciendas }\end{array}$ & $\begin{array}{c}\mathbf{N}^{\circ} \text { de } \\
\text { yanaconas }\end{array}$ & $\begin{array}{c}\text { Población } \\
\text { india total }\end{array}$ \\
\hline Omasuyos & 1786 & 169 & 20.487 & 43.075 \\
\hline Chulumani & 1786 & 336 & 18.786 & 31.004 \\
\hline Sicasica & 1792 & 206 & 17.190 & 41.542 \\
\hline Larecaja & 1786 & 270 & 14.669 & 39.946 \\
\hline Pacajes & 1796 & 90 & 8.875 & 44.777 \\
\hline La Paz & $1786-1792$ & 28 & 2.458 & 7.025 \\
\hline Total & & $\mathbf{1 . 0 9 9}$ & $\mathbf{8 2 . 4 6 5}$ & $\mathbf{2 0 7 . 3 6 9}$ \\
\hline
\end{tabular}

Fuente: H. S. Klein, Haciendas y Ayllus en Bolivia, siglo XVII y XIX.

Tabla 2: Consolidación de haciendas en el cantón de Ancoraimes 1852-1903

\begin{tabular}{|c|c|c|c|c|c|c|}
\hline $\mathbf{N}^{\circ}$ & Propietario & Propiedad & $\begin{array}{c}\text { Extensión } \\
\text { (hectáreas) }\end{array}$ & Documento & Fecha y año & $\begin{array}{c}\begin{array}{c}\text { Precio en } \\
\text { bolivianos }\end{array} \\
\end{array}$ \\
\hline 1 & $\begin{array}{l}\text { Fabián } \\
\text { Mendoza }\end{array}$ & Corpa & 3.750 & $\begin{array}{c}\text { Revisita } \\
\text { compra y } \\
\text { venta }\end{array}$ & 4/agosto/1852 & 5.900 \\
\hline 2 & $\begin{array}{l}\text { Pastora } \\
\text { Galdo de } \\
\text { Valdivia }\end{array}$ & $\begin{array}{l}\text { Corpa } \\
\text { Grande }\end{array}$ & 3.000 & Herencia & $\begin{array}{c}\text { 1/octubre/ } \\
1858\end{array}$ & 35.00 \\
\hline 3 & $\begin{array}{l}\text { Fabián } \\
\text { Mendoza }\end{array}$ & Chuspaya & 3.750 & $\begin{array}{c}\text { Revisita } \\
\text { compra y } \\
\text { venta }\end{array}$ & $\begin{array}{c}27 / \text { noviembre/ } \\
1868\end{array}$ & 7.420 \\
\hline 4 & $\begin{array}{l}\text { Elvis y } \\
\text { Asunta } \\
\text { Evia }\end{array}$ & Concahuani & 800 ha. & $\begin{array}{l}\text { Compra - } \\
\text { venta }\end{array}$ & $\begin{array}{c}\text { 18/octubre/ } \\
1879\end{array}$ & 4.400 \\
\hline 5 & & Chontamarca & & & & \\
\hline 6 & $\begin{array}{l}\text { Gregorio } \\
\text { Torrez }\end{array}$ & Huertaja & 50 ha. & $\begin{array}{c}\text { Compra y } \\
\text { venta }\end{array}$ & $\begin{array}{c}\text { 20/noviembre/ } \\
1889 \\
\end{array}$ & 250 \\
\hline
\end{tabular}

${ }^{7}$ Herbet Klein, Haciendas y Ayllus, siglos XVIII y XIX, Lima, IEP, 1995, p. 24. 
La hacienda Huanquisco Canta en manos de la familia Mendoza Iturri, provincia Omasuyos, La Paz-Bolivia

Giovana Alba Ninachoque Quispe

\begin{tabular}{|c|l|l|c|c|c|c|}
\hline 7 & $\begin{array}{l}\text { José } \\
\text { Miranda }\end{array}$ & Chejepampa & 8.400 ha. & $\begin{array}{c}\text { Compra y } \\
\text { venta }\end{array}$ & $\begin{array}{c}20 / \text { noviembre/ } \\
1889\end{array}$ & 35.000 \\
\hline 8 & $\begin{array}{l}\text { Eugenio L. } \\
\text { Orosco }\end{array}$ & Pocoata & 6.200 ha. & $\begin{array}{c}\text { Compra y } \\
\text { venta }\end{array}$ & $27 /$ marzo/1889 & 22.000 \\
\hline 9 & $\begin{array}{l}\text { Julio } \\
\text { Vallesteros }\end{array}$ & Asasillo & 4.000 ha. & $\begin{array}{c}\text { Compra- } \\
\text { venta }\end{array}$ & $27 /$ marzo/1889 & 1.600 \\
\hline 10 & $\begin{array}{l}\text { Máximo } \\
\text { Mendoza }\end{array}$ & Corpa & 2.500 ha. & Partición & $27 /$ abril/1897 & 9000 \\
\hline 11 & $\begin{array}{l}\text { Manuel L. } \\
\text { Mendoza }\end{array}$ & Chuspaya & 2.500 ha. & Partición & $27 /$ abril/1897 & 10.00 \\
\hline 12 & $\begin{array}{l}\text { Virginia } \\
\text { viuda de } \\
\text { Encinas }\end{array}$ & Suntuura & 2.500 ha. & Testamento & $8 /$ abril/1897 & 6.100 \\
\hline 13 & $\begin{array}{l}\text { Bruno } \\
\text { Burgoa }\end{array}$ & Macamaca & 7500 ha. & Ejecutorial & $13 /$ agosto/1900 & 500 \\
\hline 15 & $\begin{array}{l}\text { Faustino } \\
\text { Bozo }\end{array}$ & Sallcapampa & 1.250 ha. & Venta & $17 /$ agosto/1900 & 8.000 \\
\hline 16 & $\begin{array}{l}\text { Eugenio } \\
\text { Luna }\end{array}$ & Chinchaya & 6.200 ha. & Testamento & 22/enero/1903 & 30.000 \\
\hline 17 & $\begin{array}{l}\text { Eugenio } \\
\text { Luna }\end{array}$ & Pocoata & 5.000 ha. & Testamento & 22/enero/1903 & 4.000 \\
\hline
\end{tabular}

Fuente: Elaboración propia, en base a Lucio Tarqui Suntura. Formación de haciendas latifundios en el Altiplano central Norte del departamento de La Paz entre los años 18701940 en la provincia Omasuyos y Pacajes, Tesis de Licenciatura, 2001.

La penetración de las haciendas en el periodo republicano en Bolivia fue expandiéndose hacia el interior de las provincias en un avance continuo y rápido que buscaba agregar mano de obra. De esta forma, podemos ver claramente la consolidación de nuevas haciendas en la provincia de Omasuyos que provocó la destrucción de las comunidades indígenas. En la tabla 2 podemos observar la consolidación de haciendas en el cantón de Ancoraimes perteneciente a la provincia de Omasuyos.

En suma, podemos señalar que el proceso y formación de las haciendas en el cantón Ancoraimes de la provincia de Omasuyos se dio inicio a partir de 1852 hasta 1903, se pudo observar que cada año se estuvieron consolidando nuevas haciendas en las distintas comunidades de este cantón. Por lo que también se pudo observar que la familia Mendoza poseía haciendas en dicha región.

\section{Benjamín Mendoza Iturri y sus haciendas}

Benjamín Mendoza Iturri proviene de una familia tradicional de terratenientes del siglo XIX poderosa y rica en bienes inmuebles que llegaron a tener poder sobre las tierras indígenas en tres sectores del altiplano y los valles paceños como (Achacachi, Ancoraimes y Tímusi) estas ubicadas en las provincias de Omasuyos y Muñecas donde adquirieron una variedad de propiedades a través de herencia y compra, así mismo contaban con casas en la ciudad de La Paz ubicadas en lugares céntricos, como se verá más adelante. 
La familia Mendoza, a través de sus cinco generaciones fueron terratenientes, los datos del árbol genealógico muestran que Pedro Mendoza y Juliana Aranda, pertenecientes a la primera generación, tuvieron como descendientes a Anselmo Mendoza Aranda casado con Daria Salmon; Fabián Mendoza Aranda casado con Úrsula Sarabia; Toribio murió soltero y Rosa Mendoza Aranda se casó con José Néstor Flores, pertenecientes a la segunda generación.

La tercera generación estuvo integrada por Rosa Mendoza Salmon - hija única de Anselmo Mendoza Aranda- casada con Primitivo Agramonte; Manuel Cesar Mendoza Sarabia casado con María Rebeca Iturri; Máximo Mendoza Sarabia casado con Eusebia Flores; Epifanio Mendoza Sarabia casado con Rosa Cárdenas; y María Mendoza Sarabia casada con un hombre de apellido Portugal (se desconoce el nombre); los hijos de Fabián Mendoza Aranda también pertenecientes a la tercera generación.

La cuarta generación estuvo integrada por Ricardo Agramonte Mendoza, hijo único de Rosa Mendoza de Agramonte, estuvo casado con Rosa Moraleda; Benjamín Mendoza Iturri, Braulio Mendoza Iturri y Sara Mendoza Iturri hijos de Manuel Cesar Mendoza Sarabia; Gregoria Mendoza, hija única de Máximo Mendoza Sarabia; Luis Mendoza Cárdenas (se desconoce el nombre de su esposa); Blanca Mendoza Cárdenas (se desconoce el nombre de su esposo); Carmen Mendoza Cárdenas casada con el minero orureño José Díaz; y Jorge Mendoza Cárdenas (se desconoce el nombre de su esposa), hijos de Epifanio Mendoza Aranda; Avelino y Urbana Portugal Mendoza hijos de Rosa Mendoza Sarabia. La quinta generación conformada por Rosa Isabel Agramonte Moraleda, hija única de Ricardo Agramonte Mendoza, casada con Jorge Cusicanqui.

Grafico 1: Árbol genealógico de la familia Mendoza 


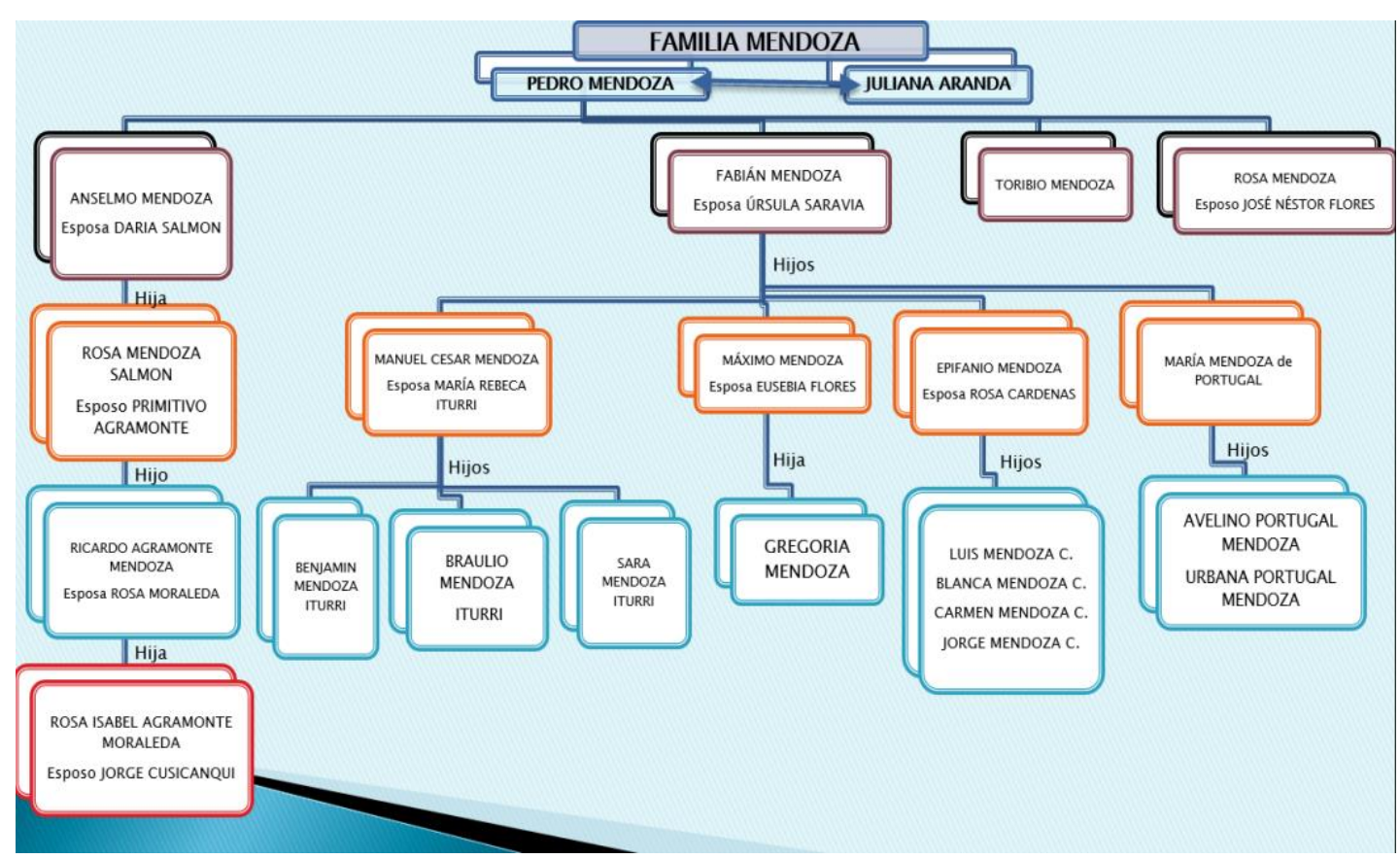

Fuente: Elaboración propia con base en la documentación del Archivo privado de la familia Mendoza.

Benjamín Mendoza Iturri, es hijo de Manuel Cesar Mendoza, fue coronel jubilado del Ejército Nacional desde 1940, propietario de múltiples propiedades rurales, en 1941 fue subprefecto de la Provincia de Omasuyos y uno de los principales fundadores del Movimiento Nacionalista Revolucionaria (MNR) en la provincia de Omasuyos, juntamente con el señor Alberto Mendoza López, primer diputado del MNR. También, participo en la Guerra del Chaco (1932-1935), fue uno de los beneméritos de la patria y como teniente coronel donde se le otorgó la medalla "Cruz de Bronce", en calidad de excombatiente de la guerra del Chaco. En 1979 falleció en la ciudad de La Paz.

Benjamín Mendoza Iturri recibió en calidad de herencia de su padre Manuel Cesar Mendoza las propiedades de "Carcapata" y "Lugaraya" situadas en el cantón de Ancoraimes provincia de Omasuyos; otra de las propiedades "Huarata" situada en Tímusi provincia de Muñecas y una tercera parte de la casa de la calle Jenaro Sanjinés ubicada en La Paz.

De igual manera, su hermano Braulio Mendoza Iturri recibió como herencia las propiedades de "Ispincuni" y "Pacayani situada en Tímusi provincia Muñecas y la propiedad "Apoco" situada en Ancoraimes provincia de Omasuyos y una tercera parte de la casa de la calle Sanjinés ubicada en la ciudad de La Paz.

En la tabla 3 podemos observar con más detenimiento las propiedades rurales y urbanas que poseía en su poder Benjamín Mendoza Iturri, quien administro hasta antes de la Reforma Agraria de 1953. 
Tabla 3: Propiedades rurales y urbanas de Benjamín Mendoza Iturri

\begin{tabular}{|c|c|c|}
\hline Propietario & Propiedades & Provincia \\
\hline \multirow{7}{*}{$\begin{array}{l}\text { Benjamín Mendoza } \\
\text { Iturri }\end{array}$} & Huanuisco Canta & \multirow{5}{*}{ Omasuyos } \\
\hline & Cohani & \\
\hline & Chuspaya-Carcapata & \\
\hline & Lugaraya & \\
\hline & Calahuancani & \\
\hline & Huarata & Muñecas \\
\hline & Inmueble urbano & $\begin{array}{l}\text { Ubicado en la calle Jenaro } \\
\text { Sanjinés, ciudad de La Paz. }\end{array}$ \\
\hline
\end{tabular}

Fuente: Elaboración propia, con los datos del testamento de Manuel César Mendoza y, en base al Expediente agrario de las propiedades de Huanquisco Canta, Lugaraya y Huarata, INRA, n 13948.

Fotografía 1: podemos observar a la familia Mendoza Iturri

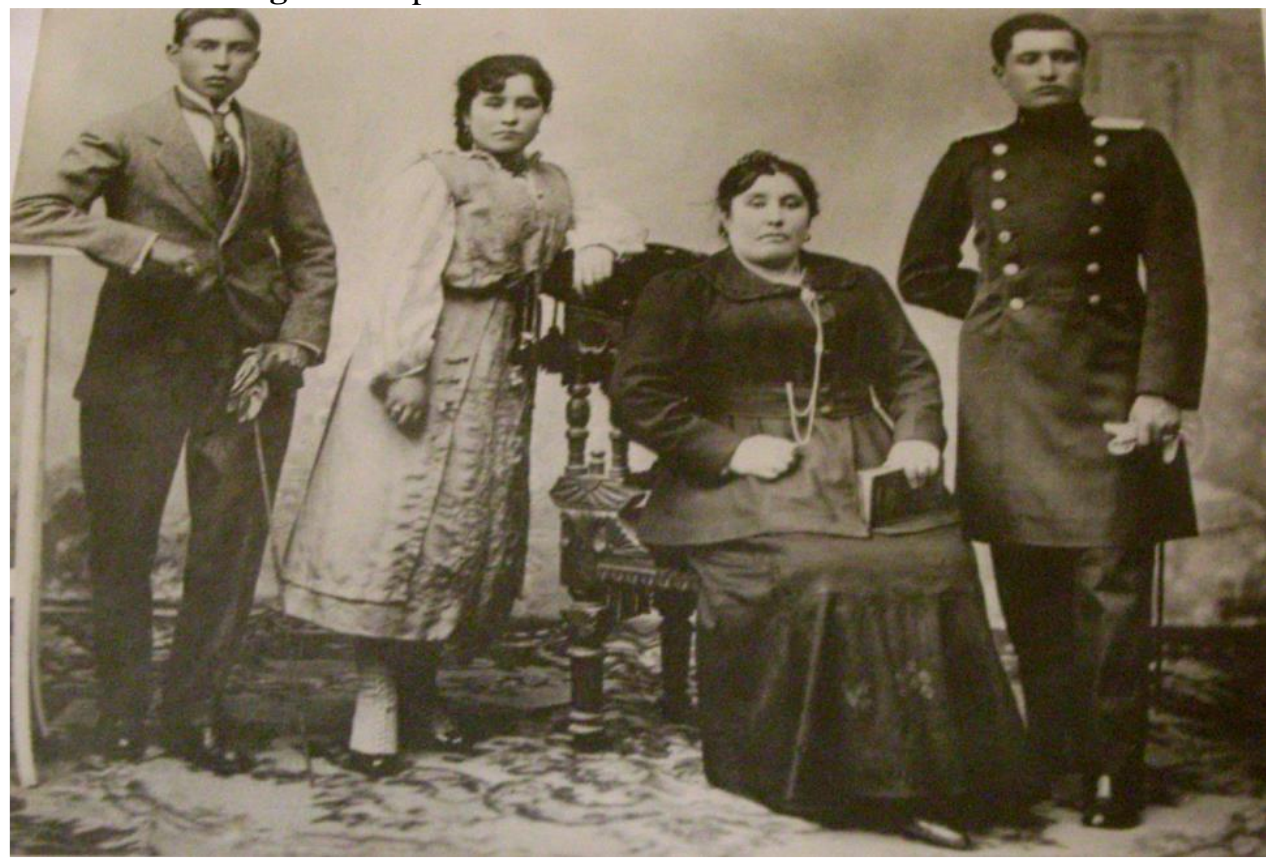

Fuente: Fotografía del Archivo privado de la familia Mendoza. En la fotografía podemos observar del lado izquierda a Braulio y a su lado a Sara Mendoza Iturri, hermanos de Benjamín Mendoza Iturri, de lado derecha observamos a Benjamín Mendoza Iturri vestido con su uniforme de coronel a su lado su madre Rebeca Iturri.

\subsection{La hacienda Huanquisco Canta}


El origen del nombre de Huanquisco Canta, según los testimonios de las personas mayores de la comunidad, "se debe a que en tiempos de los Inkas, en el lugar había una gran cantidad de pequeños conejos silvestres o salvajes (pampa wank"u) que al amanecer se escuchaba silbidos y/o bullicios de ellos (wank"u khuyusiri) como augurando malos momentos en el futuro".

Fue así que a partir del periodo prehispánico los comunarios del lugar decían que los conejos cantaban todos los días al anochecer y por ello esta comunidad había sido nombrada Wankukantani, posteriormente durante la colonia con la llegada de los españoles el nombre es castellanizado y conocido como Huanquisco Canta y registrado con la misma denominación en la etapa republicana.

La hacienda Huanquisco Canta está ubicada en el cantón Chejepampa, en la segunda sección de Ancoraimes, provincia de Omasuyos, departamento de La Paz. Limita al norte con la estancia Chiñaja, al sur con Chuspaya y Maquelaya, al este con Sulluni y Aquilambaya y al oeste con Chejepampa, camino a Ambaná por el medio. Se encuentra a una altitud de $4006 \mathrm{msnm}^{9}$, El clima generalmente es frío, los suelos son aptos para el cultivo de diversos productos, la flora es diversa en especies vegetales como, la tola, la kiswara, el pino y el eucalipto.

Con relación a la hacienda de Huanquisco Canta, hemos recurrido a fuentes primarias que nos han permitido elaborar este estudio, como los existen fuentes primarias como los padrones republicanos del Archivo La Paz (ALP/PR) y los expedientes agrarios del Instituto Nacional de Reforma Agraria (INRA). Según dichas fuentes, se puede ver que, en 1893, Huanquisco Canta era una comunidad libre y tenía como apoderado, a Alejandro Ancó registrado como el primer dueño ${ }^{10}$ de dicha comunidad.

El padrón de revisita de 1893 señala que:

Ante el señor juez, el indígena Alejandro Ancó mayor de edad casado de esta vecindad labrador y apoderado de 63 copropietarios y entre otros originarios que obediente al llamamiento legal presente el título de adjudicaciones de la ex-comunidad Canta sus límites los designados en la casilla 3ra de la papeleta 1ra su precio de cinco mil bolivianos y su mitad liquida de trescientos veinte cinco la que solicita sea constatado e inscrita el señor juez atendiendo el debido declaro por catastrado e

\footnotetext{
${ }^{8}$ Wilson Levandro Layme y Filomena Ticona Aruito, Historia de la Toponimia de las comunidades del Municipio de Ancoraimes, La Paz, Editorial Servicio Gráficos “El Clon”, 2008, p. 43.

${ }^{9}$ Véase http://es.getamap.net/mapas/bolivia/la_paz/_canta/

${ }^{10}$ Es necesario aclarar que Alejandro Ancó es sólo "apoderado". Como señala Froilán Mamani, "La ley de Exvinculación de 1874 tuvo mayor efectividad porque propuso un proceso sutil de cambios en los espacios comunales", es decir que convirtió a los comunarios en propietarios de sus tierras. En el caso de Canta, en la revisita realizada en 1893, aparece un propietario de la comunidad, registrado como apoderado para que la comunidad de Canta no perdiera su estatuto de ayllu libre. Para examinar esta cuestión con más detalle véase a Froilán Mamani Humerez, "La práctica del arrendamiento durante el siglo XIX en la región de Santiago de Huata”, en M. L. Soux, El proceso histórico hacia la territorialización del poder, p. 41.
} 
inscrita la dicha propiedad ordenando que el titulo sea anotado en el libro de resúmenes firmo el señor juez con el recojo del apoderado ${ }^{11}$.

El padrón de 1893 nos permite explicar cuatro aspectos para entender con más detalle la anterior cita:

$1^{\circ}$. Alejandro Ancó es Apoderado de 63 copropietarios, donde inscriben como proindiviso.

$2^{\circ}$. El indígena Alejandro Ancó es quien aparece en los papeles como apoderado de esta comunidad.

$3^{\circ}$. Hay un solo título de la comunidad, esto significa que esta comunidad sigue siendo una propiedad libre para esos períodos.

$4^{\circ}$. La comunidad es calificada como una propiedad e inscrita en el Catastro.

Posteriormente, para 1900, es decir siete años más tarde, Huanquisco Canta ya no era una comunidad, sino una hacienda privada de la familia Mendoza Iturri. En el expediente agrario del INRA, se encontraron informaciones sobre el hecho de que la comunidad Huanquisco Canta fue adquirida en 1900 por Manuel César Mendoza padre de Benjamín Mendoza Iturri. El documento señala:

La ex comunidad Canta de la que soy propietario por compra de los revisitarios e hijos de este, cumpliendo todas las disposiciones legales prescritas, en cada caso para la enajenación de tierra de origen se compone de las siguientes parcialidades Canta, Cohani, Lugaraya, Umamachata o Calahuancani, conforme a la matrícula de tierras y la adjudicación revisitaria está compuesta de originarios y agregados. Los tales originarios ya han fallecido totalmente y las sayañas [parcelas de tierra] han quedado vacantes pues dichos originarios no han dejado descendencia alguna legal y al igual tales originarios han abandonado sus terrenos en tiempos lejanos ya han fallecido totalmente ${ }^{12}$.

Se evidencia entonces que la comunidad Huanquisco Canta fue libre hasta 1900, y que a partir de ese año pasó a pertenecer a la familia Mendoza Iturri. El valor de la propiedad Huanquisco Canta en 1893, se puede verse en los registros catastrales de la provincia Omasuyos: Huanquisco Canta tiene un valor declarado de 5000 pesos, pero en 1936, en los registros de las Matrículas del cantón Ancoraimes, Huanquisco Canta ya tenía un valor de 40000 pesos.

Posterior, con la Reforma Agraria de 1953, las distintas haciendas sufrieron cambios, ya que a partir de ese momento éstas se empezaron a liquidar y a distribuir a los campesinos. Según el expediente agrario del INRA n ${ }^{13948,}$

\footnotetext{
${ }^{11}$ Archivo de La Paz, (La Paz, Bolivia) (ALP), Sección Republica (SR), fondo Padrones Republicanos (PR), legajo 43, folio 119

${ }^{12}$ Archivo del Instituto Nacional de Reforma Agraria, (La Paz, Bolivia) (INRA), Sección Republica (SR), Fondo Expedientes Agrarios (EA), legajo 13948, folio, 140.
} 
se puede comprobar que la hacienda Huanquisco Canta, el juzgado agrario presentó el informe correspondiente a la ex-hacienda indicando que la propiedad era en realidad un latifundio, por su considerable extensión y por el método de trabajo que se empleaba en ella. A través del informe topográfico se puso en evidencia que Huanquisco Canta tenía una extensión de 4497520 hectáreas, las cuales fueron divididos en cuatro partes.

La primera superficie, de 1120 hectáreas fue destinada para el área escolar; la segunda, de 47280 hectáreas para la estancia de Canta; la tercera de 1383340 hectáreas, para terrenos aprovechables para terrenos aynocas ${ }^{13}$; y la última de 3065780 hectáreas para el pastoreo. En el plano 1 podemos apreciar la extensión total de la hacienda Huanquisco Canta.

Plano 1: la hacienda Huanquisco Canta

\footnotetext{
${ }^{13}$ En las comunidades indígenas las aynocas, grandes extensiones de tierras, eran empleadas como parcelas colectivas, ya sea para la siembra o como lugares de pastoreo para el ganado. Véase al respecto: http://www.boliviarural.org/noticias/noticias-2010/697-campesinos-piden-distribucion-de-tierras-fiscales-de-acuerdo-consus-usos-y-costumbres.html
} 


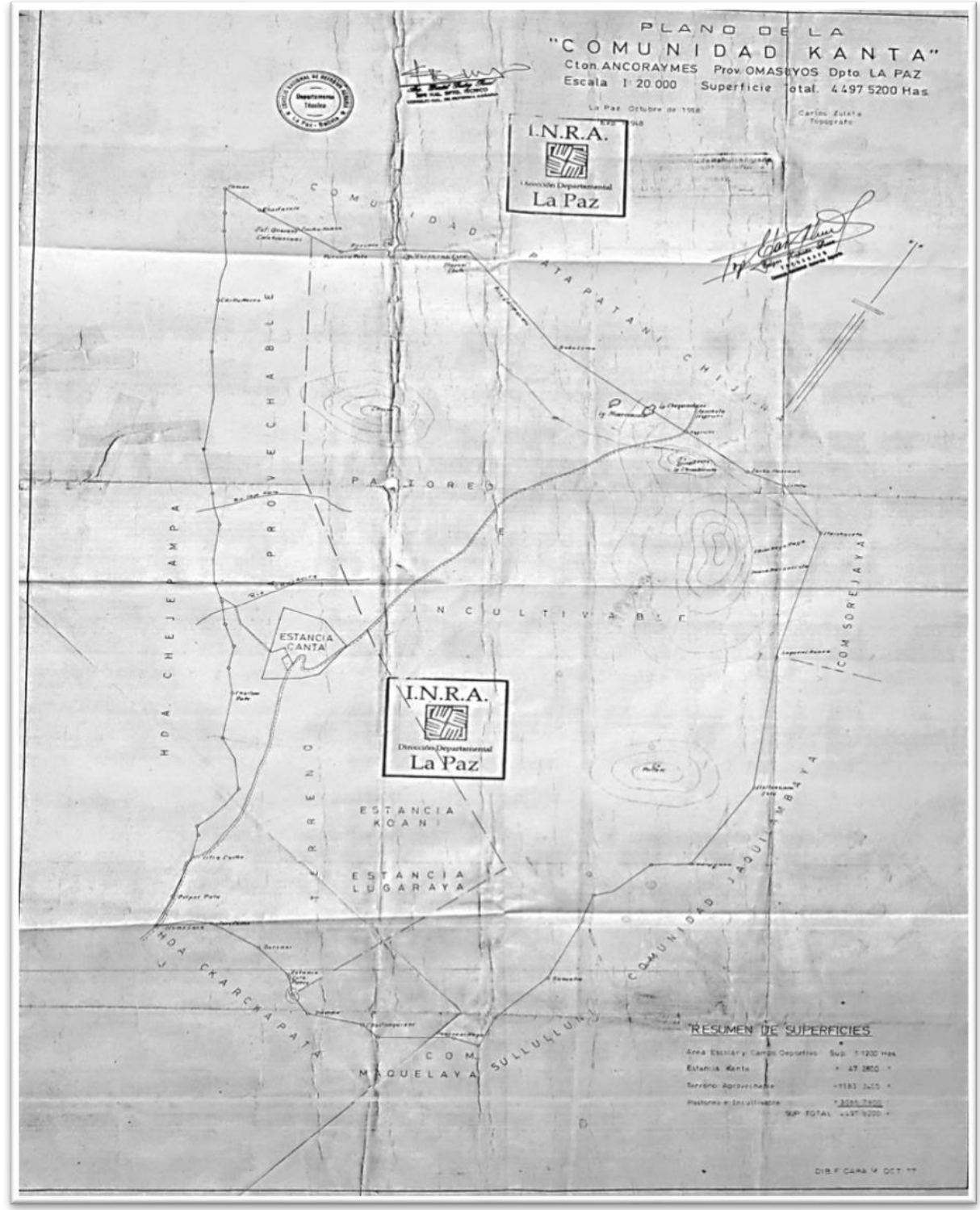

Fuente: Instituto Nacional de Reforma Agraria- Departamental, INRA, La Paz

La casa hacienda tiene una muralla hecha de piedra y cuenta con un gran número de habitaciones, tanto para el patrón como para sus colonos. Según el expediente agrario del INRA nos indica que Benjamín Mendoza Iturri había hecho construir la casa hacienda para su familia, para los colonos, dos depósitos uno para armas ${ }^{14} \mathrm{y}$ la otra para alimentos donde almacenaba todo lo que producía en la hacienda. A su vez, contaba con una pequeña capilla adornada con diversos santos hechos de piedra a quienes les rendían culto en las fiestas patronales. En la fotografía 2 se observa que aún quedan restos de la casa hacienda, la muralla de la hacienda está casi intacta. Actualmente la hacienda Huanquisco Canta, cuenta con un gran espacio, la mitad de la

${ }^{14}$ Ramón Quispe mencionó la existencia de un ambiente dedicado a guardar armamento. Entrevista realizada a Ramón Quispe ex-colono de la hacienda Huanquisco Canta, La Paz, 17 de Octubre, 2013. 
hacienda es una escuela que está al servicio de la comunidad. Así mismo los comunarios se pusieron de acuerdo para no derrumbar la casa de hacienda y que ésta quedara como un patrimonio de la comunidad.

Fotografía 2: La casa hacienda

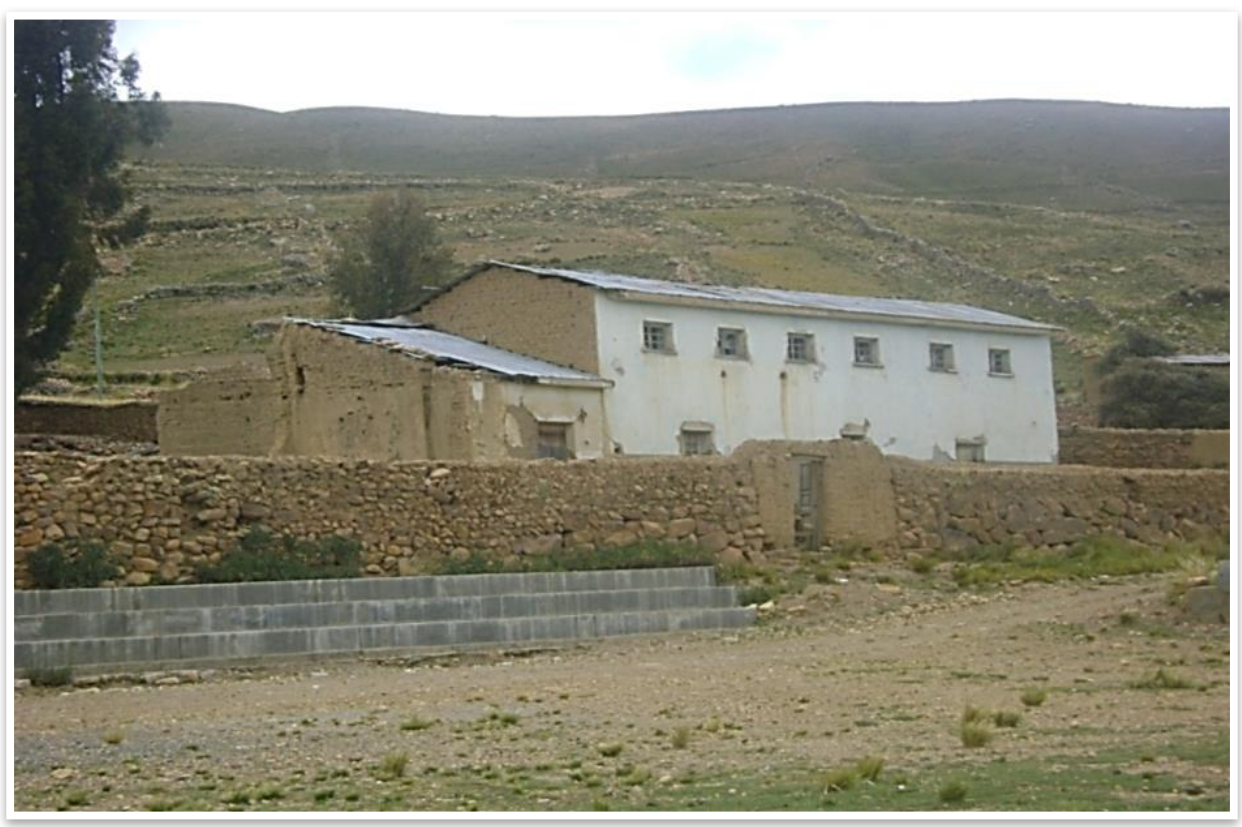

Fuente: Giovana Alba Ninachoque Quispe.

\section{Producción agrícola}

En este apartado, aunque sin la minuciosidad que hubiéramos querido presentar, se tratará de la producción agrícola de la hacienda Huanquisco Canta. Así como de la mano de obra y actividad, la inversión tecnológica y el desarrollo de la comercialización de sus productos agropecuarios hacia los centros de consumo. De forma general, las haciendas se habían convertido en uno de los elementos más importantes de la producción agropecuaria de Bolivia, entendiendo con ello que estas fueron un centro de abastecimiento de alimentos para los centros de consumo. Al respecto Danilo Paz Ballivián señalaba que en el proceso agrario antes y después de 1952:

La hacienda se vincula con el comercio de la ciudad de La Paz y algunos centros mineros ubicados en la zona, sólo a través de la venta de dos o tres productos, cereales y tubérculos [...] pero los colonos estaban excluidos de esta relación mercantil [...] el terrateniente tenía la responsabilidad exclusiva de la comercialización de la producción. Los campesinos solo participaban en los mercados rurales limitándose al trueque de sus productos dominantemente ${ }^{15}$.

\footnotetext{
${ }^{15}$ Danilo Paz Ballivián, Estructura Agraria boliviana, La Paz, Plural Editores, 2009, p. 25.
} 
Pese a la baja tecnología en el área rural, la producción en las haciendas tuvo una gran importancia dentro de la economía nacional ya que los productos eran enviados hacia la ciudad de La Paz. Por su parte, Nicolás Acosta señala que la actividad económica en el sector del altiplano de Omasuyos, Pacajes, Ingavi y Sicasica fue importante. Se cultivaban papa, papaliza, oca, quinua, alverja, haba, cebada y otros. En las regiones de Larecaja, Muñecas, Inquisivi y Cercado se producía trigo, maíz, frijoles, garbanzos, habas, hortalizas, legumbres fruta y todos los productos de valles ${ }^{16}$, y en el sector de los yungas se producían plátanos, frutas cítricas, café, cacao, coca, arroz y otros. Finalmente, los hacendados obtenían una significativa ganancia monetaria que provenía de sus haciendas, gracias a la venta de una abundante producción agrícola que provenía de sus haciendas.

Ese también fue el caso de Benjamín Mendoza Iturri, dueño de la hacienda Huanquisco Canta, quien tenía bien distribuida la tierra de su hacienda para obtener una buena producción agrícola. Una primera parte estaba destinada al pastoreo de su ganado; las demás tierras estaban sembradas con productos agrícolas.

Sin duda, la hacienda Huanquisco Canta poseía grandes extensiones de terreno, además de contar con todos los medios de comunicación para acceder a la comercialización de sus productos agrícolas, por lo que ésta se convirtió en el eje de las actividades agropecuarias. En la tabla 4 podemos distinguir, con más detalle la producción agrícola anual de la hacienda Huanquisco Canta.

Tabla 4:_producción agrícola de la hacienda Huanquisco Canta, antes de 1953

\begin{tabular}{ccc}
\hline $\mathbf{N}^{\circ}$ & Producto Agrícola & Cargas \\
\hline 1 & Papa & $3.000 \mathrm{qqs}$. \\
\hline 2 & Cebada & $150 \mathrm{qqs}$. \\
\hline 3 & Quinua & $300 \mathrm{qqs}$. \\
\hline 4 & Oca & $2.600 \mathrm{qqs}$. \\
\hline 5 & Habas & $150 \mathrm{qqs}$. \\
\hline
\end{tabular}

Fuente: Datos tomados del expediente agrario del INRA de las propiedades de Huanquisco Canta, Lugaraya y Huarata, INRA, Sección Archivo, n 13948.

La tabla 4 nos muestra que en la hacienda Huanquisco Canta se producía una variedad de papa y otros productos que se llevaban a la ciudad y a otros centros de consumo.

En la hacienda Huanquisco Canta se hacía uso de maquinarias, equipos de transporte, bueyes aradores y herramientas agrícolas ${ }^{17}$; además, esta hacienda

\footnotetext{
${ }^{16}$ Nicolás Acosta, Guía del viajero en La Paz, noticias estadísticas, históricas, locales, religiosas, templos, hoteles, edificios, antigüedades, etc., La Paz, Imprenta de la Unión Americana, 1880, p. 55.

${ }^{17}$ Entre los equipos agrícolas encontramos el arado diseñado para abrir surcos en la tierra. Este está compuesto por una cuchilla, que sirve para cortar y nivelar la tierra. En cambio la rastra es un equipo agrícola diseñado para desmenuzar las partes o porciones de tierra que han sido removidas por el arado y está compuesta por un armazón, que puede ser de madera
} 
de Benjamín Mendoza poseía un tractor de marca Macdonald ${ }^{18}$. Según el juez agrario:

El coronel Benjamín Mendoza desde que se retiró del ejército, en mil novecientos cuarenta se hizo cargo de sus fincas, moralizó a sus colonos, que antes estaban dedicados al pillaje, adquirió tractores, rastras, arados, etc. Y tres camiones y se dedicó personalmente al trabajo agrícola, de manera que Canta era una finca, donde existía gran cantidad de ganado de toda clase y productos agrícolas en cantidad por ello comercializaban ${ }^{19}$.

La comercialización de diversos productos agrícolas fue una de las principales actividades de Benjamín Mendoza; todo lo que producía en sus haciendas lo trasladaba al área urbana. En la calle Jenaro Sanjinés de la ciudad de La Paz tenía una tienda/almacén de todos los productos agrícolas que producía en sus propiedades rurales; finalmente, los vendía en la ciudad y a los centros de consumo, como las ferias rurales de Achacachi, Chejepampa, Sococoni e incluso entraba a los valles de Consata.

\subsection{La mano de obra y organización jerárquica de la hacienda Huanquisco Canta}

La hacienda Huanquisco Canta, como empresa agropecuaria, tenía una significativa cantidad de mano de obra no remunerada o en su defecto una mano de obra barata, lo que de hecho constituía uno de los factores decisivos de sus considerables beneficios. En 1900, contaba con 300 colonos; sin embargo, en 1942 solo quedaron 10 colonos.

Según, los datos del INRA sobre los colonos y mano de obra señalan que: "en el año de mil novecientos cuarenta y dos, se escaparon en masa habiendo quedado en Canta menos de diez colonos [...], el patrón contrató algunos colonos de otras fincas del contorno [...] que venían temporalmente a trabajar" 20 , dándoles un salario por el trabajo que prestaban en la hacienda en los meses de siembra y cosecha.

Por otro lado, en el juicio sobre la restitución de tierras seguido por Esteban Arratia contra Benjamín Mendoza Iturri, esté señala, "cuando yo adquirí la dicha propiedad, de la fuerza de trabajo de los cien habitantes parasitarios he hecho un núcleo de reducido de gente honrada, trabajadora y útil al país, pues

\footnotetext{
o de metal. Sobre aspectos similares en el ejercicio de la industrialización agraria, véase la tesis de Ramiro Fernández, "Cañamina: la historia de una hacienda de los yungas de Inquisivi (siglo XIX-XX)", tesis de Licenciatura en Historia, La Paz, UMSA, 1995.

${ }^{18} \mathrm{El}$ expediente agrario de la propiedad de Huanquisco Canta señala que: "El tractor de marca Macdonald \& Co, tenía las siguientes características: arado internacional (Little Genius $N^{\circ} 8-312$ ), de 3 surcos de 12; con palanca de ajuste, cuchillas circulares de 15. Alce automático, enganche para tractor con zafe automático de resorte. Mendoza Iturri lo adquirió al precio de veinte dos mil ciento cincuenta y siete bolivianos". Véase INRA, SR, EA, legajo 13948, folio, 110.

${ }^{19}$ INRA, SR, EA, legajo 13948, folio, 144.

${ }^{20}$ INRA, SR, EA, legajo 13948, folio, 110.
} 
Canta era una propiedad "progresista" donde se hacía una agropecuaria industrial" 21.

Vale la pena recordar que dentro de la hacienda Huanquisco Canta se produjo una sublevación indígena, en abril de 1953. Asimismo, Esteban Arratia, en representación de los comunarios de Canta, en el juicio mencionado señalo que el propietario de esta hacienda había cometido abusos contra los comunarios y que, a la vez, hubo una sublevación dentro la hacienda contra el propietario para que se fuese de la comunidad, pues los colonos ya estaban hartos los colonos de sus abusos y malos tratos. Así podemos verlo en la siguiente declaración:

... digan que Benjamín Mendoza es un elemento malévolo y sanguinario, conocido con el nombre de "tigre de Achacachi" y que ha victimado a los campesinos: Mariano Vallejos, Manuel Mamani, Mariano Poma, Anselmo Quispe, Benito Quenta y otros colonos más [...] declaren así mismo que Benjamín Mendoza ha violado y estuprado a las hijas y esposas de los campesinos de Canta, cuando se encontraba como propietario [...] las puertas de las habitaciones de la casa de hacienda $[\ldots]$ fueron saqueadas totalmente, por los excolonos, los que desde esos momentos se sublevaron contra el coronel Mendoza, apropiándose de todos sus ganados, que tenía en gran cantidad, asî como víveres de todo clase [...] estoy alejado de Canta desde el mes de abril de 1953, fecha del alzamiento a bala y fuego de los campesinos de Canta $^{22}$.

Nos parece adecuado señalar, que todos los colonos que trabajaban para los hacendados hasta 1953, una vez que se dictó la Ley de Reforma Agraria. Todos los colonos de la hacienda Huanquisco Canta, se enteraron de que Víctor Paz Estensoro, había dictado una ley a favor de los campesinos. Fue a partir de entonces que todos los comunarios se pusieron de acuerdo para sublevarse contra Benjamín Mendoza Iturri, cansados de sus abusos. Por tal motivo, saquearon la casa hacienda, y sacaron todo lo que había en dicha hacienda. Finalmente, cuando el patrón de la hacienda vio que sus colonos se habían sublevado en su propiedad, escapó hacia la ciudad de La Paz, pues sabía que sus propios colonos podían matarlo y desde ese momento no retornó a su hacienda.

La hacienda Huanquisco Canta está situada en un lugar de singular importancia, entre dos vías, la primera vía tiene comunicación hacia la región de Ambaná y la otra hacia la comunidad de Chuchulaya entrando a los valles de las provincias Muñecas y Larecaja. Era una de las principales e importante propiedad de Benjamín Mendoza Iturri ya que desde allí controlaba a sus demás haciendas que estaban a cargo de sus mayordomos. Por ello, la estructura social en las haciendas en las haciendas de Huanquisco Canta,

${ }^{21}$ INRA, SR, EA, legajo 13948, folio, 126.

${ }^{22}$ INRA, SR, EA, legajo 13948, folio, 135. 
Carcapata, Lugaraya, Cohani, Calahuancani y Huarata, estaba marcada por una pirámide social: en la parte superior se encontraba el dueño de la hacieda llamado patrón o terrateniente, en el segundo el mayordomo, en el tercero el jilakata y en el cuarto los colonos. En el gráfico 2 podemos observar la estructura social en la hacienda Huanquisco Canta propiedad de Benjamín Mendoza.

Gráfico 2: pirámide social en la hacienda Huanquisco Canta

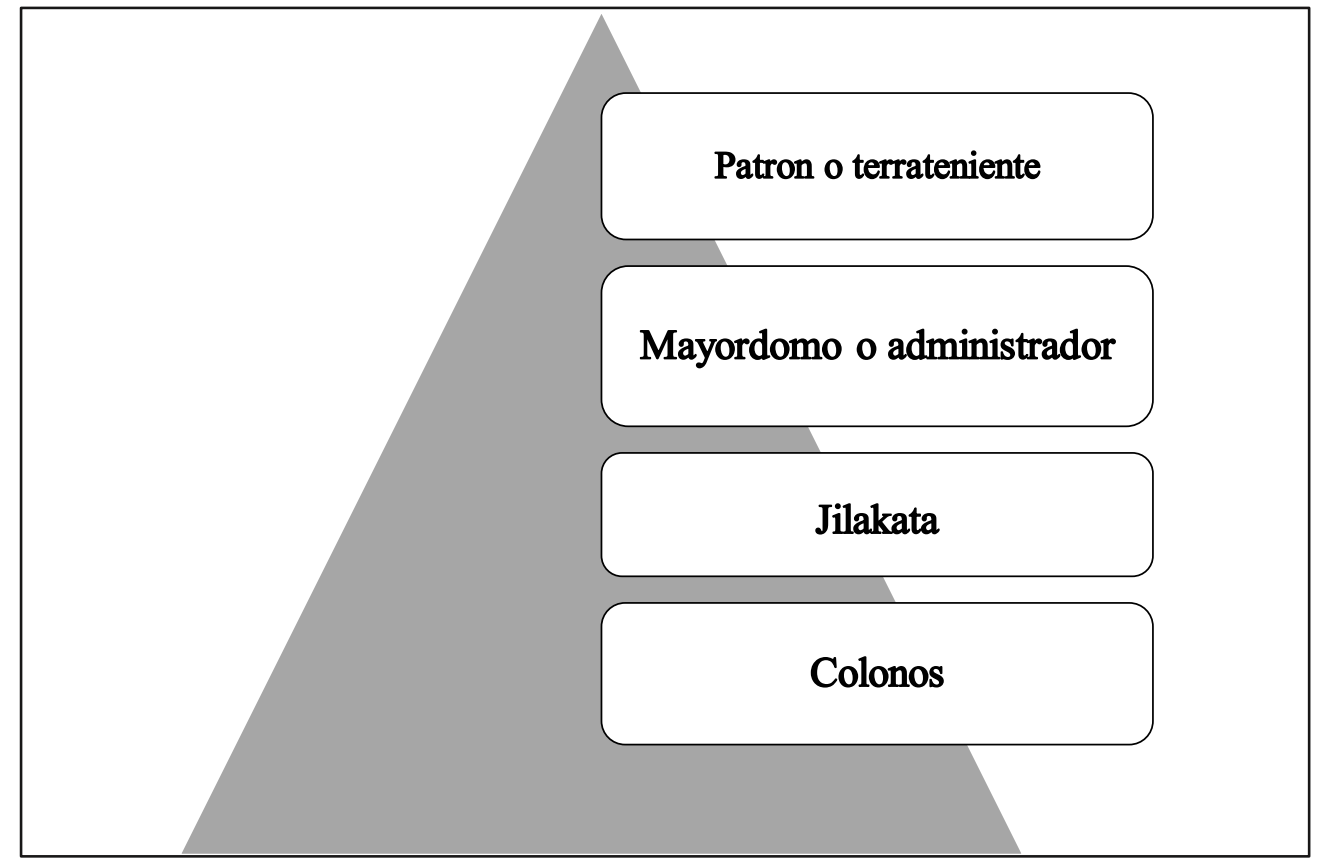

Fuente: Elaboración propia en base a las distintas entrevistas que se realizó a los ex-colonos que trabajaron en la hacienda Huanquisco Canta, 2014-2015.

El patrón era el que ocupaba el primer lugar en la jerarquía social dentro la hacienda por ello, el hacendado podía pertenecer "a un partido político como ser el liberal, conservador, republicano, radepista, emenerista, porista, pirista, o falangista" ${ }^{23}$. Los hacendados en su mayoría tenían vinculación con muchos partidos políticos, como es el caso de Benjamín Mendoza quien fue uno de los principales fundadores del MNR en la provincia Omasuyos en 1941 puesto que esta familia tenía una vinculación política con los del MNR desde los años cuarenta.

El mayordomo era mestizo o cholo, era el que cumplía y hacia cumplir los mandatos del patrón, administraba, controlaba y vigilaba todos los trabajos que él designaba a cada uno de los colonos, les manejaba con mucho poder como hacia el hacendado, era una persona generalmente de fuerte carácter que,

${ }^{23}$ Felipe Quispe Huanca, "El primer Congreso Indigenal de 1945”, tesis de Licenciatura en Historia, La Paz, UMSA, 2012, p. 27. 
... servía incondicionalmente al patrón, esta institución tenia raíces coloniales y republicanas. En ocasiones el mayordomo era mucho más avanzado que el mismo patrón, llegando a ser visto como un capataz. Entre sus funciones y obligaciones se consignaban los de hacer trabajar las parcelas de la hacienda, supervisar todo proceso de producción agrícola, así como el de hacer cumplir y respetar los servicios. Por tanto su nombramiento estaba también influenciado por los deseos del patrón" 24 .

El jilakata era un indígena de la misma comunidad designado por el patrón que estaba bajo las órdenes del mayordomo. Su función era marcar el ritmo en los tiempos de trabajo de siembra, cosecha y de hacer de intermediario entre los indígenas y el mayordomo ante el hacendado, como podemos ver a continuación:

El jilakata: en aymara quiere decir, el más crecido; era sacado de las mismas masas esclavas de la hacienda. Tenía que tener esas cualidades de fama servil, tira saco, etc. Que coordinaría con el mayordomo. Era él quien daba disciplina y castigo físico por las faltas cometidas de los colonos (ya sea grave o leve), según las normas estatutarias internas e impuestas por el hacendado. Su cargo era indefinido y su permanencia de cargar chicote dependía más que todo de la lealtad discrecional al amo patrón y demás, por la eficacia y cualidad que mostraba en hacer trabajar y administrar mejor a los indios en la hacienda ${ }^{25}$.

Mientras, el colono era un indígena que trabajaba para el hacendado a cambio que le deje vivir en su propiedad. Una de las obligaciones que tenían los colonos frente al hacendado era brindarle respeto y reverencia.

\section{A manera de conclusión}

A lo largo del siglo XIX y la primera mitad del siglo XX, las comunidades indígenas vivieron una constante lucha por no perder sus tierras de origen. Los primeros gobiernos como los de Simón Bolívar, Antonio José de Sucre, y Andrés de Santa Cruz, respetaron el ordenamiento colonial en que se reconocía la existencia de comunidades. Pero ya entrado el siglo XIX, se intensificó progresivamente el avance de las haciendas en el altiplano paceño y, durante el gobierno de Mariano Melgarejo, se organizó la venta de tierras comunales. Esto impulsó que muchos terratenientes expandieran su dominio hacia el interior del área rural, donde la producción agrícola era rentable.

Las élites terratenientes empezaron a brotar principalmente con la expansión de las haciendas desde los siglos XVIII y hasta mediados del siglo XX. Estas élites jugaron un papel importante en nuestra sociedad, donde muchos

\footnotetext{
${ }^{24}$ Tiburcio Maquera Colque, "Reforma agraria en Carabuco, 1940-1960. Consecuencia de las relaciones sociales en el proceso de la reforma agraria", tesis de Licenciatura en Historia, La Paz, UMSA, 2003, pp. 57-58.

${ }^{25}$ Felipe Quispe Huanca, "El primer Congreso Indigenal de 1945”, tesis de Licenciatura en Historia, La Paz, UMSA, 2012, p. 28.
} 
terratenientes llegaron acumular riqueza dentro y fuera de sus haciendas, en las ciudades tenían propiedades con grandes almacenamientos de alimentos agrícolas para el consumo y venta de todo lo que se producía.

Uno de los ejemplos de elite terrateniente es la familia Mendoza. Esta familia nace con los Mendoza Aranda, los esposos Pedro Mendoza y Juliana Aranda, y culminan con los Mendoza Iturri. Familia poderosa y rica en bienes inmuebles que llegaron a tener poder sobre las tierras indígenas en tres sectores del altiplano: Achacachi, Ancoraimes y Timusí, adquiriendo una variedad de propiedades a través de herencia y compra. Hay que destacar que los Mendoza se convirtieron en unos terratenientes con un prestigio social muy cotizado que permitió insertarse a muchos de esta familia a la nueva élite paceña.

Los Mendoza Iturri como terratenientes tenían los elementos definidos para poder controlar a la servidumbre que existía en sus propiedades, uno de los mecanismos importantes consistió en la disponibilidad que tenían los hacendados sobre la mano de obra, sin consulta podían disponer la expulsión, traslado o cambio de los peones. Los hacendados en sus latifundios mostraban la total concentración de su poder sobre sus colonos tomando así las decisiones de trabajo, el trato que les daba el terrateniente no le permitía tener una comunicación directa con sus colonos.

Posterior, la Reforma Agraria de 1953 trajo consiguió cambios trascendentales en lo político, económico y social. En donde, se liquidó por completo las haciendas, de tal manera se inició el proceso de afectación a las grandes y pequeñas haciendas y se hizo la distribución de la tierra a los colonos dando lugar a la formación de comunidades de ex-haciendas.

En fin, la presente tesis busca recuperar la historia de los pueblos indígenas que quedaron en el olvido, a través de una elite terrateniente, los Mendoza Iturri, que controló los espacios de Ancoraimes y Tímusi, por ello queda a la luz la historia sobre el manejo de las haciendas de la familia Mendoza Iturri que hasta antes de la ley de reforma agraria tuvo poder sobre este espacio de estudio.

\section{Bibliografía}

Acosta, Nicolás, Guía del viajero en La Paz, noticias estadísticas, históricas, locales, religiosas, templos, hoteles, edificios, antigüedades, La Paz, Imprenta de la Unión Americana, 1880.

Clark, R. J., Reforma Agraria e integración campesina la economía boliviana, La Paz, Servicio Nacional de Reforma Agraria, 1971.

Fernández Quisbert, Ramiro, "Cañamina: la historia de una hacienda de los yungas de Inquisivi (siglo XIX-XX)", Tesis de Licenciatura en Historia, La Paz, Carrera de Historia, UMSA, 1995. 
Grieshaber, P., Erwin, "Resistencia indígena a la venta de tierras comunales en el departamento de La Paz, 1881-1920”. Revista Data, 1, 1991.

Klein, Herbert, "La estructura de las haciendas a fines del siglo XIX en Bolivia: las provincias del norte del lago Titicaca". Data, 1, 1991.

Klein, Herbert, Haciendas y Ayllus, siglos XVIII y XIX, Lima, Editorial Instituto de Estudios Peruanos, 1995.

Layme, Levandro Wilson y Filomena Ticona Aruito, Historia de la Toponimia de las comunidades del Municipio de Ancoraimes, La Paz, Editorial Servicio Gráficos "El Clon", 2008.

Lofstrom, William, Radiografía de una provincia paceña Omasuyos en 1869, Sucre, ABNB, 2003.

Mamani Humerz, Froilán, "La práctica del arrendamiento durante el siglo XIX en la región de Santiago de Huata", en María Luisa Soux, (coord.), El proceso histórico hacia la territorialización del poder, La Paz, Instituto de Estudios Bolivianos, 2012.

Maquera Colque, Tiburcio, "Reforma agraria en Carabuco, 1940-1960. Consecuencia de las relaciones sociales en el proceso de la reforma agraria", La Paz, Tesis de licenciatura en Historia-UMSA, 2003.

Paredes, Manuel Rigoberto, La Provincia de Omasuyu, La Paz, Editorial Isla, 1995.

Paz Ballivián, Danilo, Estructura Agraria boliviana, La Paz, Plural Editores, 2009.

Quispe Huanca, Felipe, "El primer Congreso Indigenal de 1945", La Paz Tesis de Licenciatura en Historia-UMSA, 2012.

Rivera Cusicanqui, Silvia, "La expansión del latifundio en el altiplano boliviano elementos para la caracterización de una Oligarquía regional". Avances Revista, 2, 1978.

Rojas, Antonio, "La tierra y el Trabajo en la Articulación de la Economía Campesina con la Hacienda". Avances, 2, 1978.

Tarqui Suntura, Lucio, "Formación de haciendas latifundios en el altiplano central Norte lacustre del departamento de la paz. Periodo de 1870-1940. Provincia Omasuyos y Pacajes", La Paz Tesis de Licenciatura en Historia-UMSA, Carrera de Historia, 2001.

Urquidi, Arturo, Temas de Reforma Agraria, La Paz, Editorial Juventud, 1985. 